\title{
5
}

\section{Aspergillosis: A Disease of Modern Technology}

Aspergillosis is the least well known of the diseases we discuss in this book, in part because there is no common presentation as with ringworm and thrush, and in part because it only emerged as a serious condition late in the twentieth century. We discussed Aspergillus spores as allergens in the last chapter and in this chapter we consider its other forms. ${ }^{1}$ The first modern English language book on aspergillosis was a collection of essays published in 1985, some 20 years after that for candidiasis and 150 years after ringworm. ${ }^{2}$ The first international meeting on aspergillosis was in 1971 and was linked to concern about farmer's lung. ${ }^{3}$ Serious medical interest in aspergillosis only took off in the 1980s, with the emergence of invasive aspergillosis as an opportunistic, lifethreatening infection of immuno-compromised patients. This condition had attracted some medical attention from the 1960s as a complication of leukaemia and its profile grew with the development of high tech surgical and medical interventions, such as transplant surgery, intensive care and immunosuppressant treatment regimes. ${ }^{4}$ However, the public profile of Aspergilli fungi was much higher, not as infectious disease agents, but as producers of toxic chemicals that developed on rotting foodstuffs and introduced to the world a new class of poisons: mycotoxins. Aspergilli fungi produce compounds called aflatoxins and the detection of these prompted a debate about whether they were newly recognised, or newly produced. This ended with a consensus that they were genuinely novel and came from the unforeseen consequences of new technologies of transporting, storing and processing foodstuffs.

In this chapter, we first discuss what became known as the 'aflatoxin scare' of the 1960s, which led to the creation of a new research field of fungal toxins more widely, in large part prompted by fears that they were significant carcinogens. This episode eventually led to new standards of 
food storage and transportation across the world. Scientific and medical interest in the Aspergilli developed in the second half of the twentieth century for three reasons. Firstly, it came from the close relation of the Aspergilli to the Penicillia and technologies of antibiotic production. Secondly, the identification of various forms of aspergillosis through improved medical technologies, notably X-rays and antibiotics, which in patients with tuberculosis, literally and metaphorically opened their lungs to secondary aspergillosis infection. Finally, by the late twentieth century invasive pulmonary aspergillosis (IPA) was the most important and prevalent form of Aspergillus infection and a major problem in many medical fields. This infection can be regarded as an exemplary iatrogenic disease; it became the bane of human transplantation surgery, leukaemia treatment, and intensive care units and like candidiasis has been styled a 'disease of the diseased'.

\section{Aspergillus flavus and aflatoxins ${ }^{5}$}

In 1960, 100,000 turkeys died of a mysterious disease in southern England, mostly within 80-100 miles of London. Young birds simply collapsed in pens and fields, while adult birds showed a general malaise, with nervous symptoms, before dying. Post mortems revealed inflammation of the gut and liver damage, suggesting poisoning of some type rather than a feared contagion, such as Newcastle disease. ${ }^{6}$ The outbreak spread to ducks and pheasants, which led to major investigations by the British government's veterinary agencies and agricultural feedstuff companies, notably British Oil and Cake Mills (BOCM). The problem was not followed in detail by the press, in part because it was localised and in part, because viral fowl pest was a much larger problem at that time. ${ }^{7}$ Nonetheless, considerable efforts were made by government and corporate laboratories to find the cause of what veterinarians termed turkey ' $\mathrm{X}$ ' disease. ${ }^{8}$

Following outbreaks in other parts of the country, suspicion fell upon feedstuffs and particularly Brazilian groundnut meal that had been given to poultry for the first time in 1960. Experiments at BOCM showed that the disease could be produced by feeding Brazilian groundnut meal, however, further work at the Home Office Forensic Laboratory failed to find any specific poison. Pathological investigations at Unilever Research Laboratories, the company that was the major importer and processor of groundnuts, first showed that turkey ' $\mathrm{X}$ ' disease mainly affected the liver and, if rats were fed groundnuts for six months, some developed liver cancer. ${ }^{9}$ In December 1961, the same group announced 
that they had identified a specific poison for turkey ' $\mathrm{X}$ ' disease, a toxin produced by $A$. flavus that they called 'aflatoxin'. ${ }^{10}$ In fact, four specific toxins were soon identified, which were subsequently labelled by their fluorescent profile with marker chemicals. Those that showed blue were designated $B_{1}$ and $B_{2}$ and those that were turquoise green $G_{1}$ and $G_{2}$. Aflatoxin $B_{1}$ was shown to be the most toxic form, causing acute hepatitis, immunosuppression, and hepatocellular carcinoma. ${ }^{11}$ Research on A. flavus revealed further complexity as, rather than a single species, it turned out to be best characterised as a 'species complex'. Amongst the 11 species was A. oryzae, known in Japan as the 'kõji fungus', which rather than being associated with toxins and poisoning, had been used for centuries in the production of sake, miso and soy sauce. ${ }^{12}$ Indeed, it was termed the 'national fungus' by Professor Emeritus Eiji Ichishima of Tohoku University in the journal of the Brewing Society of Japan in $2006 .{ }^{13}$

The scare in Britain attracted considerable interest across the world and led to the development of a new field of research on mycotoxins. In the case of aflatoxins, investigators found the toxin in food imports sourced from East and West Africa and possibly India. A number of experimental studies showed that liver disease and liver cancer could be produced in a number of animal species, but the threat to human health remained unproven. An editorial in the British Medical Journal in February 1962 warned against any panic over foodstuffs imported from developing countries, but called for improved monitoring and more research on liver disease in countries where groundnuts and similar foods were dietary staples. ${ }^{14}$ The following year, aflatoxin was found in peanuts in the United States, which when fed to ducklings produced the characteristic disease syndrome and cancers. ${ }^{15}$ The NIAID began to fund screening programmes to find and identify mycotoxins. ${ }^{16}$ While there remained no direct evidence of a threat to human health, in part because any exposure was likely to be sporadic and at low doses, reports of cancer from fungal metabolites grew. Amongst the most worrying were those from 'antibiotic species': for example, liver tumours in rats fed rice infected with Penicillium islandicum and, to the dismay of dermatologists, mice fed griseofulvin. ${ }^{17}$ In 1965, the FDA introduced a standard for safe aflatoxin levels in foodstuffs of 30 parts per billion in peanut products, with levels above that requiring action. This policy set in chain new research projects on methods of monitoring and changes to regulatory responsibilities. ${ }^{18}$

The discovery of aflatoxin led to what was soon coined 'the mycotoxin Gold Rush', as researchers across the world investigated a whole 
new class of poisons and their health effects. ${ }^{19}$ The American Society for Microbiology held a session on the subject at its annual meeting in July 1965 and other conferences followed. Why was so much effort and so many resources mobilised to meet 'a seemingly obscure toxicity syndrome in poultry flocks'? ${ }^{20}$ First, doctors and food producers feared that aflatoxin was the tip of a toxin iceberg and that the long-term effects of fungal poisons had been overlooked. Reviews suggested that aflatoxins might have been the cause of previously unexplained outbreaks of disease in guinea pigs in 1954, dogs in 1955, and cats and rabbits in $1957 .{ }^{21}$ There was also the possibility that other fungi might be contaminating different foodstuffs, with most concern about those imported from developing countries, where standards of husbandry and storage were suspect. In addition, the conditions and time taken in transporting products with the development of international markets meant there were more opportunities for deterioration and, hence, the production of toxins. Thus, there was impetus from state agencies and the public for scientists to develop a better understanding of the problem, and new methods to test and monitor for toxins. The globalisation of the food industry had created a completely new threat to human and animal health.

A second factor stoking interest was the fear that mycotoxins were possibly major causes of cancer; hence, new technologies of food production, storage and transportation might have been contributory factors to an increase in the incidence of the disease in the twentieth century. ${ }^{22}$ The 1960s saw growing awareness of the long-term effects of chemicals in the environment, as the warnings of Rachel Carson's Silent Spring finally hit home, and as we discussed in Chapter 3, the 1960s and 1970s saw the development of clinical ecology and its warnings of direct and indirect environmental threats to human health. These concerns had focused on man-made chemicals, but now there was a further twist; new technologies were turning foodstuffs and natural products into sources of danger. Liver cancer was known to have a distinctive geographical distribution and had become a model for field studies of the causes of cancer. ${ }^{23}$ The disease was most common in subSaharan Africa and Denis Burkitt, already famous for his identification of what became Burkitt's lymphoma, highlighted the possible impact of aflatoxin in his survey of cancers in East Africa in $1965 .{ }^{24}$ In 1966, Richard Doll, well known for his work linking cigarette smoking and lung cancer, estimated that liver cancer rates in developed countries could be cut 10 to 15 -fold, if people were able to reduce their exposure to carcinogens. ${ }^{25}$ 
At the end of the 1960s, John Higginson, a leading cancer epidemiologist, reviewed what might be possible in terms of prevention from all causes, in an analysis that divided aetiology into four categories: cultural, occupational, miscellaneous and unknown. ${ }^{26}$ Unsurprisingly, lung cancer was 90\% cultural. Colon and rectal cancers were 99\% unknown. Liver cancer was $40 \%$ cultural and 60\% unknown. With the latter, Higginson associated aflatoxin with DDT as possible triggers, but conceded that, though there was experimental evidence of carcinogenesis in animals, there was insufficient evidence of a danger to human health and he saw no case for tightening regulations. ${ }^{27}$ While epidemiological studies remained inconclusive, the potency of aflatoxins as carcinogens made them useful agents for laboratory studies, particularly by inducing hepatomas in rats and rainbow trout. ${ }^{28}$

In 1979, one review of the burgeoning research on aflatoxins in the previous decade observed, 'Unfortunately the amount of factual information seems to be out of all proportion to the amount of light shed on the particular mechanisms by which aflatoxins damage susceptible cells. ${ }^{29}$ A similar conclusion with regard to safety policy was reached in a review by a committee constituted at the Institute of Medicine in Washington, and published in 1979 by the National Academy of Sciences (NAS). ${ }^{30}$ The context was concern about whether saccharin, the most commonly used artificial sweetener, was a carcinogen and aflatoxins, along with mercury and nitrites, were included as comparators. ${ }^{31}$ The review was very controversial. It recommended that food safety policy be made 'simpler, more flexible and more comprehensible', and that regulatory agencies be granted greater 'discretionary authority'. The suggestion was that the FDA could allow 'small amounts of carcinogenic additives in the food supply as it sees fit'. The review also suggested that an assessment of health risk versus economic benefit was necessary, plus an evaluation of the comparative risk of different foods. ${ }^{32}$ But who would undertake the work and how risks were to be judged was left open.

The research trajectory with regard to the necessity for risk assessment with aflatoxins exemplified the problems of translating science into policy. Firstly, the link between the toxicity of aflatoxins in animals and humans was uncertain, and it had proved impossible to establish tolerance levels. Secondly, it seemed likely that there should be different safe levels for different foodstuffs, and that this would have to take into account secondary effects, for example, human exposure to milk from cows fed contaminated grains. Thirdly, the sporadic incidence of aflatoxin contamination, along with the potentially high price 
of prevention, meant that benefits seemed unlikely to be worth the cost. Finally, the number of stakeholders involved meant that it had proved very difficult and time consuming to have changes approved; for example, the five-year delay in reaching a decision on the 1974 FDA proposal to reduce the tolerance level of aflatoxin from 20 parts per billion (ppb) to $15 \mathrm{ppb}$.

The NAS review was framed within the overall context of the decline in deaths from infectious diseases, the rise in those from cardiovascular disease and cancer, and the changes to the human environment brought by technological development. ${ }^{33}$ In the specific area of food, the authors pointed to technical advances having shifted hazards 'away from microbial contamination that produced acute disease soon after exposure', to 'chemicals in small quantities and other hazards introduced by environmental contamination as well as by food production and processing.$^{34}$ The recommendation for regulatory flexibility came from the aim of allowing agencies to find a middle way between unrestrained use and a ban, as well as a desire to allow them to keep up with progress in research, production and monitoring. ${ }^{35}$ In other words, the problem of aflatoxins was being defined largely in terms of the risks created by modern methods of food production, distribution and consumption; nonetheless, it was expected to be solved by those very same technologies. ${ }^{36}$ One factor, echoing issues with regionally specific fungi, was that risks varied with place and individual lifestyles, particularly dietary, meaning that national, let alone international, standards were likely to be hard to agree and even harder to enforce. ${ }^{37}$

\section{Aspergillosis: 'A Rare Disease', 1900-1960}

Poultry were important in the story of Aspergilli fungi and human health at both ends of the twentieth century, as aspergillosis was recognised as a lung disease of fowl and cattle, and an occupational disease in humans around $1900 .{ }^{38}$ Veterinarians reported the disease to be most common in birds, including chickens, turkeys and certain waterfowl, where it caused pneumonia and other lung diseases. ${ }^{39}$ Mammals were said to be less susceptible, but it was reported in cattle and dogs. Veterinarians blamed contaminated grain, but argued that certain predisposing conditions were necessary, such as the animals being in poor general health or living in insanitary conditions. 'Brooder pneumonia', an American term for aspergillosis, came from its presence in intensively reared fowl. In humans, the disease was found mainly in those who worked with birds and cattle, those who handled grain or worked in dusty conditions. The primary presentation in humans was as bronchial or lung 
disease, though it was also reported sometimes to affect the cornea of the eye and the middle ear. The classic accounts of the disease came from Paris and its prevalence amongst 'les gaveurs de pigeons', the men who force-fed the birds being reared for food. ${ }^{40}$ They did so by mixing grain and water in their mouths, chewing the mixture to a pulp and then spitting it forcibly down the gullet of the birds. This practice was undertaken on an industrial scale, with each man allegedly feeding 2,000 birds each day.

Pulmonary aspergillosis attracted the attention of doctors because of the similarities of its symptoms and pathology with pulmonary tuberculosis, and over whether it was a cause of a disease known as 'pseudo-tuberculosis'. ${ }^{41}$ The great anti-tuberculosis campaigns, which began across Europe and North America at the end of the nineteenth century, brought more patients with lung diseases into the medical gaze, and doctors were required to differentiate those who would benefit from the new initiatives in treatment and care, such as those to be offered the sanatorium treatment. ${ }^{42}$ There was also unhappiness that 'pseudo-' diseases still existed in an era when developments in pathology had led to greater specificity and aetiological constructions of disease. The Pathological Society of London formed a Committee on Pseudo-tuberculosis in 1899 and its report recommended that the name be dropped from the medical lexicon and that only lesions caused by the Koch's bacillus be termed tubercles. All other pulmonary lesions were to be referred to as 'nodules', one class of which was 'aspergillar nodules'. ${ }^{43}$

The first book on Aspergillosis was published in France in 1897 by Louis Rénon, who was Chef de Clinique a la Faculté de medècine de Paris. ${ }^{44}$ Renon's work was one inspiration for an MD thesis on Aspergillosis submitted to the Victoria University of Manchester by Thomas Rothwell in 1899. ${ }^{45}$ Rothwell's supervisor, Sheridan Delépine, Professor of Pathology, had published on Aspergillus skin infection 1894 and was active in tuberculosis research. ${ }^{46}$ Rothwell reviewed the literature on the disease and experimental work on the fungus, before describing his own studies on the inoculations of spores into guinea pigs. ${ }^{47}$ There had been work in Germany by Paul Grawitz, who was interested in immunity and the question of why a seemingly saprophytic organism became pathogenic; he asked: was this due to a change in the fungi, or the resistance of the host organism? ${ }^{48}$ Louis Renon's 300-page book on, what he acknowledged was, 'a rare condition' discussed the disease in animals and in the laboratory, before considering 'Aspergillose de l'homme'. ${ }^{49}$ With human disease, his main concern was whether infection was primary or secondary, and he concluded that it could be both, with $A$. fumigatus 
the most pathogenic species. Rothwell's thesis had similar conclusions, concentrating on the differences between $A$. fumigatus and the more benign $A$. niger.

Humphry Rolleston, a leading London physician who specialised in pulmonary tuberculosis, published an account of pulmonary aspergillosis in $1898 .^{50}$ He stated that it was almost exclusively a trade disease amongst millers, agricultural labourers, and others who worked with contaminated grains and with processes that created dust. He was clear that both $A$. fumigatus and A. niger could also infect the ear and skin, and wondered how many people diagnosed as suffering from pulmonary tuberculosis might really have aspergillosis, or have the fungus and bacillus acting synergistically. ${ }^{51}$ The symptoms of pulmonary aspergillosis - a cough, purulent expectoration, coughing blood, bronchitis, consolidation at the top of the lung and raised temperature - were similar to those of pulmonary tuberculosis, which left the microscopic examination of sputum as the only means for differential diagnosis. As a primary infection, the prognosis with aspergillosis was 'less grave' than for pulmonary tuberculosis, but as a secondary complication it was said to be very serious and 'in fact a terminal complication'. ${ }^{52}$

In the inter-war period aspergillosis disappeared from the medical gaze; and when it was discussed it was to admit ignorance, especially of its relation to pulmonary tuberculosis. ${ }^{53}$ Instead, the period witnessed great interest in the use of the Aspergilli in human food production, building on knowledge from Japan. The Aspergilli became a prime interest of industrial chemists, they were termed 'cell factories', because of their role in the production of citric, gluconic, itaconic and kojic acids, in what would now be termed biotechnology ${ }^{54}$ From 1917, A. niger was the mainstay of citric acid production, using technologies that were later adapted for the production of penicillin. ${ }^{55}$ Scientists at Pfizer, then a fine chemicals business, with industrial as well as pharmaceutical products, had developed large-scale methods to meet the demands of the rapidly growing soft drinks and processed food industries. ${ }^{56}$ In the 1930s, fermentation research was developed at the Industrial Farm Research Division (IFRD) of the United States Department of Agriculture. For example, in 1937 scientists from the Division published a paper setting out how to increase the efficiency of gluconic acid production with $A$. niger in submerged cultures. ${ }^{57}$ This biotechnological application of the Aspergilli directly benefited the wartime initiatives with the new antibiotics, for example scientists from the IFRD moved to the Northern Research Laboratory at Peoria when it was established in December 1940 to work on 'deep culture' production of penicillin. ${ }^{58}$ Thus, the link 
between Aspergilli and antibiotics was not, as with Candida, that new drugs opened the body to secondary fungal infections, rather it was the vital role the genus played in the production of antibiotics and hence the development of modern pharmaceutical industry.

As discussed in Chapter 3, the discovery of penicillin began a search for new fungal-derived antibacterials and the botanical similarities between Penicillium spp. and Aspergillus spp. meant that the latter became prime targets. Selman Waksman had first explored the antibacterial substances produced by A. fumigatus in the 1940 s. $^{59}$ He identified a compound that he called 'fumigacin', which had an antibacterial spectrum similar to penicillin, but was weaker and never developed for clinical use. No doubt expecting similar products to penicillin, researchers coined the terms 'Aspergillins' to refer to the range of antibiotic substances derived from the Aspergilli. The most successful of the compounds was aspergillic acid, which had some effect against Mycobacterium tuberculosis. ${ }^{60}$ Indeed, for many years Aspergillus spp. and Streptomyces spp. were regarded as most likely to produce anti-tuberculosis drugs, and, of course, the latter eventually yielded streptomycin. ${ }^{61}$

While mycologists and industrial biologists worked intensively on the fungus, aspergillosis continued to be largely ignored by doctors and the only publications were on 'rare' and 'unusual' cases of invasive disease. The Second World War gave no military, social or epidemiological stimulus to the incidence or profile of aspergillosis, as there had been with ringworm and troop invalidism, Candida and the spread of antibiotics, and migration and the geographically specific mycoses. In the United States, John Downing and Norman Conant in their 1945 review of mycotic infections for the New England Journal of Medicine did not mention aspergillosis. ${ }^{62}$ Eight years later, in his review in the same journal, Otis Jillson was cursory on the topic, noting several recent reports where aspergillosis was usually caused by $A$. fumigatus and that there was no effective treatment. ${ }^{63}$ However, he pointed to the difficulties with diagnosis, even at autopsy, because $A$. fumigatus was such a common contaminant in pathological and bacteriological laboratories, and hospital buildings.

In Britain, aspergillosis was discussed in detail in James Duncan's fungal disease survey in 1945, in relation to both pulmonary disease and farmer's lung. ${ }^{64}$ With pulmonary disease, cases were few and far between, and difficult to diagnose because of confusion with tuberculosis. However, in the 1950s a new disease entity was recognised: Aspergillus fungus balls, or aspergilloma. ${ }^{65}$ They were first reported by 
doctors in France, seen in chest X-rays from patients with cavities in their lungs left by healed tuberculosis lesions. This was a growing group due to the effectiveness of triple antibiotic therapy, with streptomycin, para-aminosalycilic acid (PAS) and isoniazid. However, the growths were not just found in former tubercular patients, they were also associated with other chronic lung conditions, of which there was growing awareness and investigation; for example, histoplasmosis and cavitating lung cancer. ${ }^{66}$ A survey in 1968 by the Research Committee of the British Thoracic Association looked at 544 patients with persistent lung cavities larger than $2.5 \mathrm{~cm}$ in diameter. ${ }^{67}$ Using X-rays, they found $11 \%$ of patients showed evidence of aspergilloma, with a further $4 \%$ probable cases. They also tested their blood serum for Aspergillus precipitins, which revealed $25 \%$ of the group were positive, though half these patients showed no X-ray evidence of infection. A follow-up study of the same group of patients, published in 1970, revealed that the presence of an aspergilloma did not increase mortality ${ }^{68}$ The important factor in mortality was that aspergilloma patients had worse coughs and were more liable to serious complications, such as haemoptysis. In around $10 \%$ of patients the aspergilloma had disappeared spontaneously, which led to the conclusion that treatment was, perhaps, unnecessary because they were benign, saprophytic growths. Where treatments were given, the procedures were similar to those developed for tubercular lesions. Thoracic surgeons, especially in the United States, removed the infected part of the lung (resection), while physicians used antifungal drugs. Only amphotericin B was effective and given by intra-cavity injection into the lung. ${ }^{69}$ By the 1980 s treatment had become more conservative, with surgery reserved for patients with severe haemoptysis. ${ }^{70}$

In the 1990s, aspergilloma was subsumed into the class of chronic pulmonary aspergillosis (CPA), which also included chronic cavitary pulmonary aspergillosis (CCPA), where patients had cavities without fungal balls, and chronic fibrosing pulmonary aspergillosis (CFPA), which developed when a primary infection remained untreated and scarred the lungs. In most cases, patients with CPA were found to have an underlying disease, typically tuberculosis, ABPA, lung cancer, COPD, emphysema, asthma or silicosis. As such, CPA typified the view that, like invasive candidiasis, aspergillosis was a 'disease of the diseased'.

\section{Invasive aspergillosis}

In the 1960s, the incidence of invasive candidiasis was much higher than that of invasive aspergillosis, but this changed in the next half 
century. Looking back from 1965, Samuel Asper and Andrew Heffernan reviewed the numbers of those diagnosed with aspergillosis in Johns Hopkins University Hospital from 1941 to $1963 .^{71}$ Their work was prompted by the aflatoxin scare and based on a re-examination of the autopsy reports of 26 cases, to determine if patients with aspergillosis had also suffered from liver disease. ${ }^{72}$ They found no correlation, but determined to extract something useful from their data they turned their attention to aspergillosis in general, responding to reports that it was on the rise. At Johns Hopkins, its incidence had increased, but only slowly and it was still relatively rare. In the 23 years surveyed, there had been 26 cases, amongst 202 patients with fungal infections identified at autopsy. The total number of autopsies was 14,819 , so the incidence of recognised fungal infection, which was no doubt an underestimate, was quite low at $1.3 \%$. However, there was a marked recent increase in aspergillosis, with 23 cases occurring in the ten years up to 1963 (Figure 5.1).

The authors regarded their most interesting finding as the association of aspergillosis with leukaemia, the incidence of which had nearly

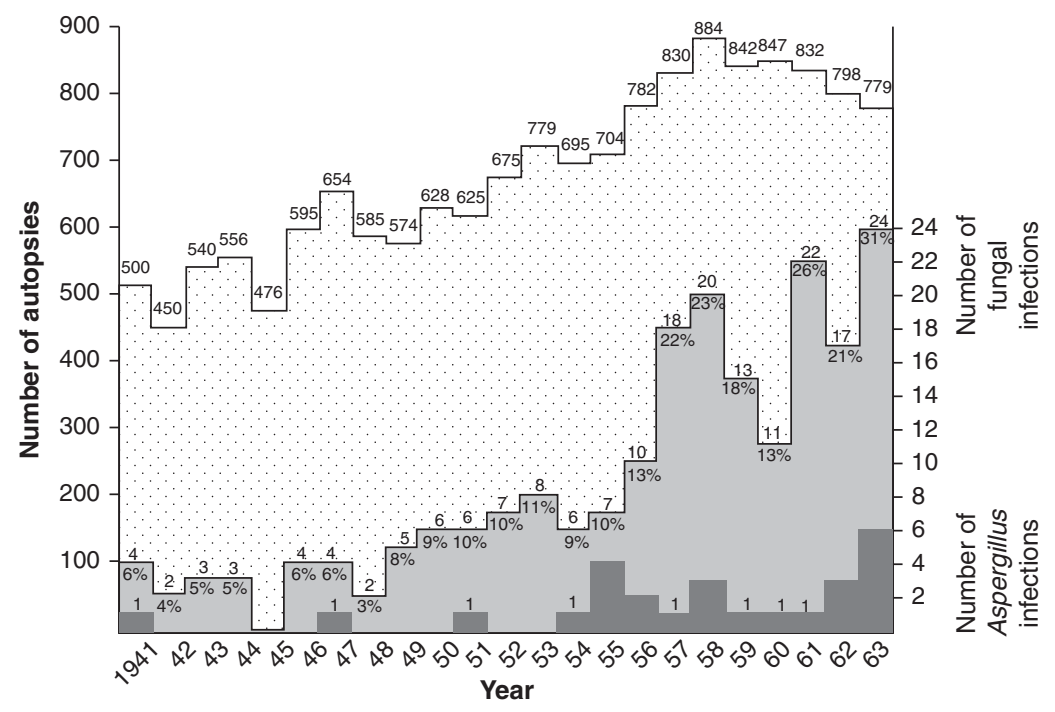

Figure 5.1 Incidence of fungal infections (including aspergillosis) found at autopsy at the Johns Hopkins Hospital, 1941-1963. ${ }^{73}$ Asper, S. P. and Heffernan, A. G. A., 'Insidious fungal disease', Trans Am Clin Climatol Assoc, 1965, 76: 101. This figure (C) 2013 American Clinical and Climatological Association used under Creative Commons Attribution - Non-commercial licence: http:// creativecommons.org/licenses/by/3.0/ 
doubled since the 1940s, during a period when treatments had intensified. ${ }^{74}$ Asper and Heffernan observed,

It may well be, as others have suggested, that unique forms of therapy for leukemia, which alter host-parasite relationships, are the factors responsible for the increasing incidence of aspergillosis. In the weeks before death, all the leukemic patients had received antibiotics and steroids and all but one had received cytotoxic agents. ${ }^{75}$

They did not speculate on which of these treatments might be responsible for predisposing patients to aspergillosis, or the extent to which the leukaemia itself was a factor.

Other doctors had already speculated on the role of fungal infections in leukaemia deaths. Two years previously, in 1963, John Gruhn and John Sansom, of the Mount Sinai Hospital, Chicago, wrote that many studies suggested 'that the profound leukopenia [decrease in the number of white blood cells] induced by antileukemic drugs appeared to increase susceptibility to infections' ${ }^{76}$ A literature review found reports of mycoses in leukaemia patients at between 14\% and 30\%, while their own retrospective look at 25 autopsies found a $24 \%$ incidence in the period 1941-1961, increasing to $39 \%$ in the last five years. In total, candidiasis was found in 19 patients and aspergillosis in eight. ${ }^{77}$ The authors concluded that leukopenia, antibiotics and steroids were all factors in fungal infections, though suggested that antibiotics were more important with candidiasis, and steroids with aspergillosis. ${ }^{78}$ This conclusion chimed with earlier studies by Herschel Sidransky in New Orleans, who had explored the relative importance of steroids and antibiotics in experimental studies of aspergillosis with mice. ${ }^{79}$

The evidence mounted that the rise in aspergillosis cases was largely due to the rise in leukaemia cases. ${ }^{80}$ In 1970, Robert C. Young and colleagues published on the pattern of aspergillosis found in the 98 patients autopsied at the Clinical Centre of the NIH between 1953 and $1970 .{ }^{81}$ Leukaemia and lymph node cancers accounted for $86 \%$ of cases, with lung disease the main clinical manifestation. Aspergillosis had only rarely been diagnosed ante mortem. Three years after the NIH report, Richard Meyer and colleagues at the Memorial SloanKettering Cancer Center, published a study that showed the prevalence of aspergillosis in leukaemia patients was rising quickly, such that in the first half of 1971, ' 41 per cent of the patients who died with acute leukemia had evidence of aspergillosis'. ${ }^{82}$ A study published in 1972, also from the Mount Sinai Hospital, New York, reported that amongst 
65 leukaemia patients: 16 had candidiasis, aspergillosis and four phycomycetes (common moulds). ${ }^{83}$ Their finding chimed with those in other hospitals, where fungal complications ranged from $14-30 \% .{ }^{84}$

The increase in the prevalence of fungal infections associated with leukaemias led doctors to seek better diagnostic methods and treatments, especially new ways to use amphotericin B, the only antifungal effective against $A$. fumigatus at this time. ${ }^{85}$ In cases of IPA, looking for increased levels of the fungus in sputum was an obvious diagnostic test, however, as the infection was deep seated in cavities and even contained within an aspergilloma, it was possible that very few fungi would reach the throat or mouth. Indeed, sputum tests were regarded only as indicative, because of the ubiquity of the Aspergilli in the environment, in hospitals, and in the sputum of healthy individuals. The alternative test was for $A$. fumigatus antibodies in blood serum. The test used so-called 'precipitins', that is, proteins or other antigenic material from the fungus that would combine with antibodies to produce a cloudy precipitate when mixed with serum. This test had problems of specificity and sensitivity, again because of the presence of $A$. fumigatus in the environment and because of variable reactions to different antigens. ${ }^{86}$

From the early 1970s, aspergilloma was counted as a type of IPA, though it was identified and treated at outpatient chest clinics and framed, alongside pulmonary tuberculosis as a public health problem. In April 1970, the British Medical Journal published an article on the incidence of systemic mycoses in Britain, based on diagnoses made by the Public Health Laboratory Service in the week ending 3 April 1970, which was said to be typical. ${ }^{87}$ Aspergilloma and aspergillosis were by far the largest reported infections: 225 cases of aspergilloma were due to A. flavus, 19 to other Aspergilli, and 15 to all other fungi. As discussed in Chapter 4, the Aspergilli loomed large amongst allergens, but the number of patients affected was below that with C. albicans. The report again emphasised that fungal diseases were neglected conditions, but the authors wanted to demonstrate that laboratory testing was available and that aspergillosis should be on clinicians' radar.

In the 1960s a new patient group emerged as potential sufferers of invasive aspergillosis - renal transplant recipients. The first modern kidney transplant, between identical twins, was performed by Joseph Murray in California in $1954 .{ }^{88}$ As the donor and recipient were genetically and immunologically identical there was no problem of organ rejection. The number of operations between identical twins and close relatives increased, with Murray leading the field in the management 
of immunosuppression with local irradiation, specific cytotoxic drugs, initially Azathioprine, and corticosteroids (presnidone). The first renal transplant operation in Britain was also between identical twins, by Michael Woodruff in Edinburgh in 1960. By the mid-1960s, Murray and a growing cadre of transplant specialists had developed regimes to better match tissue types and to manage rejection and other complications, which enabled transplants between individual of similar tissue-type and this led the number of renal transplant operations to increase rapidly. Over the same period, the median survival time of recipients rose, with those receiving organs from live patients doing better than those receiving cadaver kidneys.

The major problem in the early years of renal transplantation was rejection, but as this was better managed and recipients lived longer, other complications came to the fore, principally cardiovascular disease, osteoporosis and infection. The most important infection was with cytomegalovirus, which affected the liver and lungs, and pneumocystis pneumonia. A significant minority of recipients developed mycoses due to a variety of opportunistic fungi: cryptococcosis, candidiasis and aspergillosis. ${ }^{89}$ With aspergillosis, infection rates were relatively low, but mortality rates were high because of the difficulties with diagnosis and treatment. However, clinicians' awareness of the problem led to the introduction of prophylactic measures, for example, better surgical hygiene and patient care to prevent opportunistic infection and the pre- and post-operative use of antifungal drugs. A particular problem with renal transplant was that the most effective therapy for invasive aspergillosis was amphotericin B, where nephrotoxicity was the major side effect. Aspergillosis was also a problem in heart transplant patients; indeed, the condition reached the pages of the national press in 1969 when Britain's third heart transplant patient, Charles Hendrick, died four months after his operation from aspergillosis. The press described aspergillosis as a 'very rare' infection and one that Hendrick's doctors took a long time to diagnose, ultimately giving amphotericin $\mathrm{B}$ too late to save his life. ${ }^{90}$

Amphotericin B had the reputation amongst some doctors of being a treatment that was worse than the disease; it was often kept back for so-called 'salvage therapy' - a final attempt at therapy in the terminally ill or palliation. ${ }^{91}$ However, in 1973, Bill St Clair Symmers, a pathologist at the Charing Cross Hospital and Medical School, complained of 'pharmacophobia' amongst physicians about amphotericin B. ${ }^{92} \mathrm{He}$ discussed five cases of invasive fungal infection, none was aspergillosis, where the drug was not used because of fears of nephrotoxicity 
and the patients died. He argued that the patients should have been given the chance of overcoming the infection and that dialysis might have been used 'to tide the patient over in the event of severe renal damage', or that 'the loss of both kidneys is not necessarily fatal or incompatible with a useful life nowadays' ${ }^{93}$ This view received support from Ross Forgan-Smith and J. H. Darrell of the Royal Postgraduate Medical School, who argued that, provided doses were kept low and blood urea monitored, the drug was safe and that kidney damage was often reversible. ${ }^{94}$ One reason why physicians were reluctant to use amphotericin $\mathrm{B}$ was that diagnosis remained difficult. It seemed that many of those advocating its use were pathologists and medical mycologists, who had the benefit of post mortem evidence, which had not been available to clinicians. Despite its problems, amphotericin $\mathrm{B}$ remained the first choice therapy for invasive aspergillosis into the 1980s and was valued because it was also effective against other opportunistic fungal infections that might be present, such as candidiasis and cryptococcosis.

The primary site of aspergillosis remained the lung and inhalation the main route of infection, thus, chest physicians and infectious disease clinicians, who saw most patients of this type, became the experts on the disease. One issue that remained vexing was whether aspergillosis was always a secondary infection, with opinion shifting to the view that it could be primary in patients whose health was poor due to heart or lung problems. Better diagnosis revealed, or perhaps improved treatments produced, a new type of disease - chronic pulmonary aspergillosis (CPA). The term was first used in the late 1970s to differentiate patients with infection from those with chronic allergic reactions, but as noted above was adopted for all types of chronic infection, including aspergilloma. ${ }^{95}$

\section{Azoles again}

The story of invasive aspergillosis in the last quarter of the twentieth century was dominated by the development, trialling and clinical use of new antifungal drugs. The biggest change in preventive and therapeutic possibilities came in the 1980s with the introduction of triazole antifungal drugs. These compounds, of which the most promising were itraconazole and fluconazole, were produced from the series that began with clotrimazole. ${ }^{96}$ Itraconazole was found in a programme of screening by Janssen Pharamceutica in Belgium. ${ }^{97}$ The compound was first used in trials in 1982 and was given final approval in Britain in 1989 and the United States in 1992. ${ }^{98}$ The momentum behind the search for new antifungals was evident in the fact that an international 
symposium on itraconazole was held in Oaxaca, Mexico, as early as October 1985, a full four years before its first regulatory approval. ${ }^{99}$ Fluconazole enjoyed the same treatment, with Pfizer Pharmaceuticals sponsoring a symposium on early trials in Dorado Beach, Puerto Rico, in October 1988. ${ }^{100}$ There were hopes that the drug would be valuable against oropharyngeal candidiasis in AIDS patients and this excited public and medical attention. ${ }^{101}$ However, fluconazole's promise with aspergillosis was not realised. ${ }^{102}$ Nonetheless, it was valued because of its broad-spectrum activity, with both superficial and systemic mycoses, and convenient oral administration. ${ }^{103}$ In an article in the Journal of Antimicrobial Chemotherapy in January 1987, Roderick Hay had noted the great potential of triazole compounds because their targets were not well covered by existing antifungals. ${ }^{104}$ However, he added that 'It will be a challenge to devise suitable methods of establishing their therapeutic roles in systemic fungal disease.'

Itraconazole was valuable with aspergillosis and prompted the first ever international symposium on 'Aspergillus and aspergillosis' ${ }^{105}$ Held at the University of Antwerp in June 1987, and jointly sponsored by ISHAM and the Janssen Research Foundation, the event attracted 145 participants from 25 countries. The proceedings were co-edited by two Janssen Research Foundation scientists and Donald Mackenzie, who worked at the Mycological Reference Laboratory in London. All aspects of aspergillosis, allergic, toxigenic and pathogenic, were discussed, but the focus was on invasive disease and its treatment, with the majority of papers on treatment and prophylaxis with, unsurprisingly, itraconazole. The number of laboratories and clinicians that had been given the drug for trials was quite large. An underlying thread in the meeting was that amongst the general medical profession, aspergillosis and other fungal infections did not have the profile their prevalence and severity deserved. Indeed, delegates worried that the perception across medicine was that medical mycologists and clinicians were again exaggerating the importance of fungal infections to bring attention and resources to their area of specialist work. ${ }^{106}$ One example was the problem of fungal infection in hospitals where building works were in progress. A lot of publicity had been given to how such works could be life threatening to immunosuppressed patient because of the liberation of fungal spores, yet infection control specialists had dismissed this as small beer in comparison to the dangers of hospital acquired bacterial infections, such as MRSA.

In his concluding comments at the Antwerp Symposium, Heinz Seeliger, Director of Institute for Hygiene and Microbiology at the University of Würzburg, suggested that itraconazole appeared to have started 'a 
new area of chemotherapy'. ${ }^{107}$ Such hopes were soon to prove premature and several issues arose. First, it proved difficult to find sufficient cases for conclusive clinical trials, both to secure regulatory approval and to evaluate the new drug against amphotericin B. ${ }^{108}$ When itraconazole finally passed all the trial stages and gained FDA approval in 1992, it was essentially as a 'salvage therapy'. Second, one of the alleged advantages of itraconazole was oral administration. However, Janssen found it hard to produce a formulation that was absorbed well enough to deliver a consistent effective dose. ${ }^{109}$ Also, the active principle had to be protected from stomach acids, which meant patients had to swallow a large, coated capsule. Evidence also emerged in the early 1990s of a relatively large number of conflicts with other drugs, especially those likely to be taken by immuno-compromised patients. At the same time, new forms of amphotericin B were developed to mitigate its toxicity, mostly by binding the drug to lipids to avoid kidney damage, but these innovations were not taken up rapidly as they were not supported by randomised trials. ${ }^{110}$ Trials with invasive aspergillosis were problematic because the number of patients were not only small, but were dispersed across the country, had different forms of the infection in different regions of the body, and they suffered from a wide range of underlying conditions. ${ }^{111}$

Nonetheless, by the mid-1990s, whilst still limited, treatment options for invasive aspergillosis had improved. Clinicians had gained better understandings of drug actions, more choices and protocols for different sites and types of infection. It was still the case, however, that many patients with the disease were diagnosed too late for effective therapy, though on this front there were promising new approaches from molecular biology for earlier and more accurate detection. One hope was that improved and standardised methods would make the recognition of infection easier for non-specialists. A lot of research was undertaken on antigen tests, but a breakthrough came with microassays to detect sugars released by growing Aspergillus hyphae. ${ }^{112}$

The profile of invasive aspergillosis grew because of its high mortality and the growing number of patients affected due to the greater use of immunosuppression with transplant, cancer and other patient groups. ${ }^{113}$ Aspergillosis was an emerging infection in intensive care units, where monitoring and maintenance technologies, along with more aggressive therapies, opened the body to opportunistic infection. There was evidence in the mid-1990s from the United States and Germany that it had replaced candidiasis as the principal hospitalacquired fungal infection. ${ }^{114}$ 
A report on the changed picture and growing problem of invasive aspergillosis was presented in a European Science Foundation in 1998. ${ }^{115}$ The summary gave range of incidence in different patient populations with health compromising underlying conditions at up to one in four for leukaemia patients and around one in eight for transplant recipients. (See Table 5.1): By comparison, the rate of infection amongst ordinary patients was less than 1\%. A similar report by the Aspergillus Study Group in the United States, published in Medicine in 2000, based on data from 595 patients collected from 89 physicians, found a similar pattern. ${ }^{116}$ At this time, the main treatments remained amphotericin B and itraconazole, separate or in combination, which still gave complete cures in only $27 \%$ of cases. The conclusion of reviews at this time was, as it had been for decades, that 'new approaches and new therapies are needed to improve the outcome of invasive aspergillosis in high risk patients' ${ }^{117}$

There was, however, another new triazole in the pipeline voriconazole. ${ }^{118}$ This was another antifungal drug from the Pfizer research laboratories at Sandwich in Kent. It was produced from their programme to improve the effectiveness and range of triazoles. ${ }^{119}$ The compound, first named UK-109,496, was subject to intensive in vitro testing in the 1990s, with new experimental methods and protocols used in comparative tests against amphotericin B and itraconazole. ${ }^{120}$ Trials with mice and guinea pigs showed voriconazole to be an effective, broad spectrum antifungal, with high activity against Aspergillus spp. ${ }^{121}$ Clinical trials showed that voriconazole had advantages over existing

Table 5.1 Incidence of invasive aspergillosis according to underlying condition. ${ }^{122}$

\begin{tabular}{lr}
\hline Condition & Range (\%) \\
\hline Lung transplantation & $17-16$ \\
Allogeneic bone marrow transplantation & $5-15$ \\
Acute leukaemia & $5-24$ \\
Heart transplantation & $2-13$ \\
Pancreas transplantation & $1-4$ \\
Renal transplantation & $0.5-10$ \\
AIDS & $0-12$ \\
Multiple myeloma (stage III) & $\sim 4$ \\
Severe combined immunodeficiency & $\sim 4$ \\
Solid tumour and lymphoma & $\sim 1-3$ \\
Autologous bone marrow transplant & $\sim 1$ \\
Connective tissue diseases & $\sim 1$ \\
\hline
\end{tabular}


therapies in three areas: antifungal activity, administration and side effects. The drug was approved in the United States in May 2002 and in Europe the following year, primarily for the treatment of invasive aspergillosis. Its superiority over amphotericin B was celebrated in the Lancet in October 2002, though the advantages were relative: just over half of patients with invasive aspergillosis responded to voriconazole compared to just under a third on amphotericin. ${ }^{123}$ Nonetheless, clinicians continued to be cautiously optimistic, not just about this drug, but because it pointed the way forward to more and better antifungals. ${ }^{124}$

Aspergillosis remains a rare disease, though in the first decade of the twenty-first century it became the largest cause of death from fungal infection in Britain and was second only to candidiasis in the United States. ${ }^{125}$ Its impact in medicine was significant as it became associated with high profile settings: 'high tech' medical and surgical sites, particularly cancer and transplantation clinics, intensive care units and in the many clinical encounters that used immunosuppressant therapies. In one sense, aspergillosis was an exemplary iatrogenic condition an unintended result of medical progress. ${ }^{126}$ Indeed, the history of aspergillosis in the twentieth century can be mapped on key concerns and innovations. Pulmonary aspergillosis first attracted attention in the context of new interest in pulmonary tuberculosis around 1900 and re-emerged with mass X-ray and effective antibiotic treatment in the 1940s. Aspergilli spp. were also part of the rise of allergy and asthma, the development of antibiotics, and the discovery of mycotoxins. The profile of the infection was in step with larger epidemiological changes in mortality and especially morbidity: the decline in infections and the rise of cancers and other degenerative conditions, where chronicity and aggressive treatments opened the body to opportunistic infection. Serious disease was most common in a new type of patient - the posttransplant, immuno-compromised patient, who had been created and had to be maintained by modern medical technologies.

Except where otherwise noted, this work is licensed under a Creative Commons Attribution 3.0 Unported License. To view a copy of this license, visit http://creativecommons.org/licenses/by/3.0/ 